Revista Arbitrada Interdisciplinaria KOINONIA

Año 2020. Vol V. N 4 . Especial: Contabilidad

Hecho el depósito de Ley: FA2016000010

ISSN: 2542-3088

FUNDACIÓN KOINONIA (F.K). Santa Ana de Coro. Venezuela.

Elva Johanna Belduma-Belduma; Daniel Jacobo Andrade-Pesantez; Pablo Barahona-Vásquez

http://dx.doi.org/10.35381/r.k.v5i4.953

\title{
Decisiones de producción en el sector bananero y su relación con el flujo de efectivo
}

\section{Production decisions in the banana sector and their relationship with cash flow}

\author{
Elva Johanna Belduma-Belduma \\ elva.beluma37@est.ucacue.edu.ec \\ Universidad Católica de Cuenca, Cuenca \\ Ecuador \\ https://orcid.org/0000-0001-9333-9588 \\ Daniel Jacobo Andrade-Pesantez \\ idandradep@ucacue.edu.ec \\ Universidad Católica de Cuenca, Cuenca \\ Ecuador \\ https://orcid.org/0000-0003-0586-4038 \\ Pablo Barahona-Vásquez \\ pbarahona@ucacue.edu.ec \\ Universidad Católica de Cuenca, Cuenca \\ Ecuador \\ https://orcid.org/0000-0003-1743-0392
}

Recibido: 01 de junio de 2020

Revisado: 20 de junio de 2020

Aprobado: 02 de septiembre de 2020

Publicado: 24 de septiembre de 2020 


\section{RESUMEN}

La investigación tuvo por objeto elaborar una guía para el diseño del flujo de efectivo que aporte a la toma de decisiones de producción en las empresas del sector bananero del Cantón el Guabo. Se adoptó un tipo descriptiva con diseño de campo no experimental transversal. Un $75 \%$ de los participantes afirmó que consideran sustancial la información del flujo de efectivo para la toma de decisiones. Los resultados son consistentes con la necesidad de aplicar una herramienta eficaz que evalué el disponible de las bananeras y considerar que el flujo de efectivo es el estado financiero más viable como apoyo del departamento contable en sus funciones de reportar a los directivos. La guía propuesta del estudio cumple con las consideraciones de la Superintendencia de Compañías y normas contables que facilita al profesional de contabilidad una secuencia de pasos para elaborar con facilidad el flujo de efectivo.

Descriptores: Empresa; toma de decisiones; contabilidad; guía de estudios. (Palabras tomadas del Tesauro UNESCO).

\section{ABSTRACT}

The purpose of the investigation was to develop a guide for the design of cash flow that contributes to production decision-making in companies in the banana sector of Cantón el Guabo. A descriptive type with a non-experimental cross-sectional field design was adopted. $75 \%$ of the participants affirmed that they consider the cash flow information to be substantial for decision making. The results are consistent with the need to apply an effective tool to evaluate the availability of banana companies and consider that cash flow is the most viable financial statement to support the accounting department in its functions of reporting to managers. The proposed study guide complies with the considerations of the Superintendency of Companies and accounting standards that provide the professional accountant with a sequence of steps to easily prepare the cash flow.

Descriptors: Enterprise; decision making; accounting; curriculum guides. (Words taken from the UNESCO Thesaurus). 


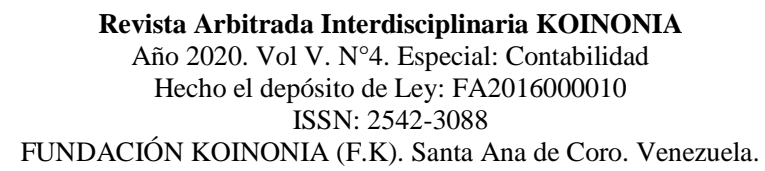

Elva Johanna Belduma-Belduma; Daniel Jacobo Andrade-Pesantez; Pablo Barahona-Vásquez

\section{INTRODUCCIÓN}

En el mundo de los negocios, las reservas de efectivo inadecuadas se convierten en una de las principales razones por la que ciertas empresas perecen en el intento de buscar el éxito; la falta de una buena administración de recursos que no consideran fundamental para sus objetivos. Con la aplicación de nuevas normas y procedimientos contables, en el Ecuador los flujos de efectivo, son uno de los informes financieros por excelencia, solicitados en cada periodo por el ente rector a las compañías, bajo las Normas Internacionales de Información Financiera (NIIF), a fin de supervisar las actividades y buen funcionamiento de las entidades económicas, y que facultan a los administradores, de las pequeñas, medianas y grandes empresas de los sectores económicos de la región, como herramientas de análisis y control financiero, por la información relevante que suministra de la capacidad operacional del negocio, para generar o maximizar el efectivo a largo plazo (Ortíz-Anaya \& Ortíz-Niño, 2018).

El sector bananero del país, es una de las fuentes económicas más representativas e importantes, por cuanto constituyen el $10 \%$ de las exportaciones agrícolas totales, y con las mayores tasas de crecimiento, sin embargo, los gremios de productores de banano, se han encarecido de políticas, normas y procedimientos para definir funciones específicas y lineamientos a seguir, para dar cumplimiento a las reformas contables e impidiéndose la implementación de herramientas que fortalezcan el crecimiento de sus negocios. En este punto, una de las mayores repercusiones que manifiestan ciertas empresas, es la idea errónea, que los informes manuales, no sistematizados, son útiles para informar de la situación financiera del negocio; en tanto los instrumentos financieros, representa pérdida de tiempo, sustentándose en datos empíricos, que no demuestra la capacidad real operativa del negocio, para asumir obligaciones, reinvertir ni adquirir financiamientos. (Urgal-González \& García-Vázquez, 2006).

El incumplimiento de las metas planteadas, es el resultado final de la falta de implementación de herramientas eficaces como el flujo de efectivo, para conocer la 


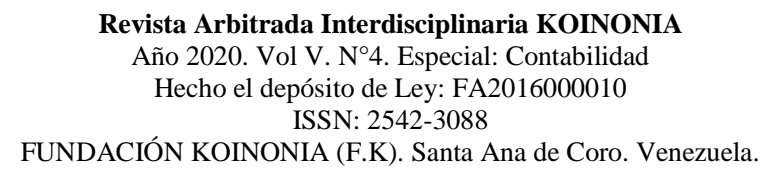

Elva Johanna Belduma-Belduma; Daniel Jacobo Andrade-Pesantez; Pablo Barahona-Vásquez

capacidad adquisitiva del negocio, y evitar el incremento de niveles de endeudamientos. Bajo este antecedente, se plantea la problemática a resolver ¿cuál es la contribución del flujo de efectivo en la toma de decisiones de producción del sector bananero?

La importancia de la problemática se hace presente ante el desasosiego de los productores bananeros, dado el desconocimiento general de las funciones operativas del negocio que aporte a la administración de los rubros económicos de mejor manera en cada fase del ciclo productivo del banano y la dificultad para implementar estrategias administrativas-productivas.

\section{Referencial teórico}

\section{El estado de flujo de efectivo como herramienta de análisis empresarial}

Investigaciones relacionadas con el sector comercial, de servicios, agropecuarios en departamentos de Colombia, y enfocándose en las actividades de operación, financiación y de inversión, determina el paralelismo con variables macroeconómicas como la tasa de empleo, producto interno bruto, tasa representativa del mercado con los flujos de efectivo (Escobar-Arias, 2016); (Escobar-Arias, 2018). Empresas colombianas con recursos limitados, para un proceso adecuado de valoración financiera de sus unidades, recurren a la metodología de los flujos de efectivo, por la confiabilidad que representa, basándose en la capacidad de las empresas al mantener actividades económicas de forma continua, generar ingresos futuros y flujos de efectivo, independientes del tamaño, actividad y sector al que pertenezca (Andrade-Valenzuela, et al., 2018); (Tovar-Posso \& MuñozMartínez, 2018).

La estructura financiera en el caso de empresas españolas está en función del nivel de endeudamiento, y la relatividad surge a partir de los flujos de efectivo, donde cuanto mayor sea el flujo de circulante en el ejercicio, mayor será la confianza de las instituciones financieras para acceder a planes de financiamiento e inversión que aporten a estrategias de la gerencia adoptadas en materia de adquisición de maquinarias, suministros de 


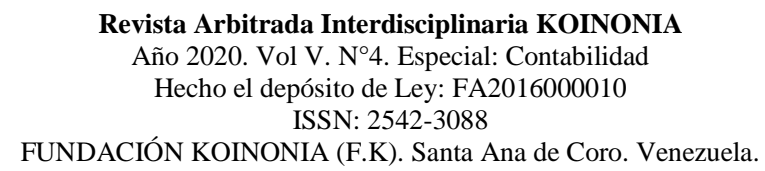

Elva Johanna Belduma-Belduma; Daniel Jacobo Andrade-Pesantez; Pablo Barahona-Vásquez

trabajo, infraestructura de las instalaciones y negociaciones en otros ámbitos (RodríguezMasero \& López-Manjón, 2016).

Para la preparación del flujo de efectivo, se necesita analizar conceptos para una mejor comprensión y análisis de la incorporación de esta herramienta financiera en las decisiones corporativas y los indicadores inherentes (Funes, 2012). Según (Ortíz-Anaya \& Ortíz-Niño, 2018) el flujo de efectivo es la reconstrucción de las entradas, salidas y saldo final de los movimientos del efectivo, originados en un período económico que evidencia los rubros de los que se originaron el efectivo, los gastos incurridos ya sea para la adquisición de bienes, materiales y equipos necesarios para que se realice sin contratiempo las actividades operativas.

La implementación de esta herramienta en las actividades ayuda a conocer si el negocio cuenta o no con liquidez disponible para tomar decisiones; y será preferible elaborarlo en periodos mensuales o semanales, puesto que entre más corto sea el tiempo los datos serán más exactos y fiables. (ApoloOrdoñez, et al., 2019).

Por otro lado, (Rodríguez-Masero, et al., 2016) bajo un contexto más simplificado, manifiestan que el flujo de efectivo refleja la diferencia entre el número de unidades monetarias, el número de entradas que salió en una actividad económica y, representa uno de los informes más sustanciales que se obtiene a partir de los estados financieros. El estado de flujo de efectivo dentro de la visión integral contable constituye un documento valioso por la información de los rubros de efectivo que se ponen en manifiesto, dada la relevancia que tiene para medir y acrecentar la solvencia del negocio; sin embargo, (Vargas-Soto, 2007) señala que el estado financiero se apoya en suposiciones que no garantizan la validez de la información vertida y por tanto carece de confiabilidad para el directivo de una organización. En este sentido, se considera esencial contar con los anexos de trabajo que garantice información real de los movimientos financieros generados en el giro del negocio y disipe la incertidumbre que se produzca en el entorno para cubrir obligaciones. 


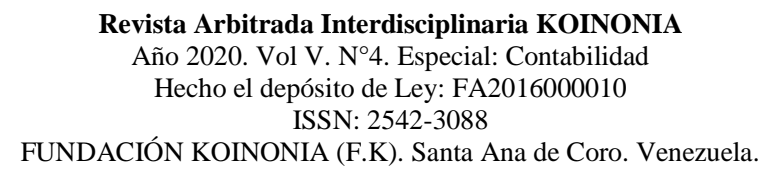

Elva Johanna Belduma-Belduma; Daniel Jacobo Andrade-Pesantez; Pablo Barahona-Vásquez

De acuerdo con la NIC 7 la información gestada por el estado de flujo de efectivo es útil, porque suministra una base para evaluar la capacidad de un negocio de generar dinero y en que necesidades está empleándose, distribuidos en tres categorías específicas: actividades de operación, de inversión y financiamiento (Van-Horne \& Wachowicz, 2002). Cuando el flujo de efectivo se emplea junto con los demás estados financieros aporta a los directivos de las empresas una visión más clara de la situación financiera del negocio, que permite evaluar la capacidad del ente económico para asumir deudas adquiridas, identificar la necesidad de financiamiento externo y los efectos de las inversiones y endeudamientos.

La NIC 7 indica que hay dos métodos de presentación del estado de flujo de efectivo, donde el método directo consiste en una reestructuración del estado de resultados integrales mediante una clasificación de las entradas y salidas del efectivo. Tiene la ventaja de la comprensibilidad y fácil distribución de los rubros que informa, relacionado con las actividades operativas del negocio. También, aporta como un instrumento de proyección para hacer comparaciones del comportamiento del efectivo en periodos específicos determinándose decisiones significativas (Gonzalo-Angulo, 2003). El método indirecto consiste en la preparación de una conciliación a partir de la utilidad neta del ejercicio y el flujo de efectivo de las actividades de operación, donde se suman y restan los ajustes para dejarlo en términos del efectivo y se colocan las variaciones de las cuentas del activo y pasivo corriente, relativas a la operación del ente; excluyéndose las cuentas de inversiones y de obligaciones financieras (Duque-Sánchez, 2015). Es más compleja su comprensión, sin embargo, aporta información más productiva para analizar la política financiera y contable de la empresa.

Si bien es cierto, ambos modelos arrojan información valiosa para el análisis posterior de la situación operacional del negocio, el método directo tiene mayor relevancia para decisiones acertadas de las fluctuaciones más remanentes de un negocio. El flujo de efectivo permite a la empresa consolidarse en el entorno económico; así como terminar en un quiebre inminente cuando se omite el control y administración del recurso más 


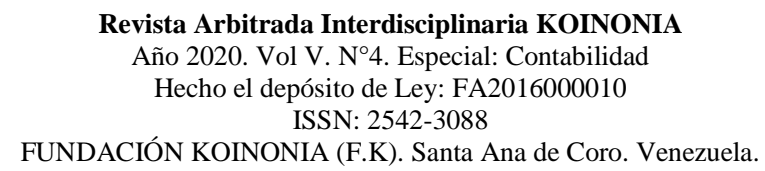

Elva Johanna Belduma-Belduma; Daniel Jacobo Andrade-Pesantez; Pablo Barahona-Vásquez

delicado en un negocio como el efectivo, con la necesidad de recurrir a capital propio o aumentar los niveles de endeudamiento no factibles para la empresa; por tanto, es indispensable la elaboración de informes como el flujo de efectivo para el buen desenvolvimiento de las actividades comerciales (Saavedra-García \& Loé-Uribe, 2018). La presentación del estado de flujo de efectivo depende de las actividades de cada organización y en el periodo que resulte más conveniente, diario, semanal o mensual, dependiendo de las necesidades de la administración; en tanto que, la información que se suministra permita evaluar la posición financiera de la entidad e inclusive evaluar el proceso de actividades ejecutadas en el entorno. En la figura 1 se presentan los tipos de actividades que se consideran en la estructura del estado de flujo de efectivo.

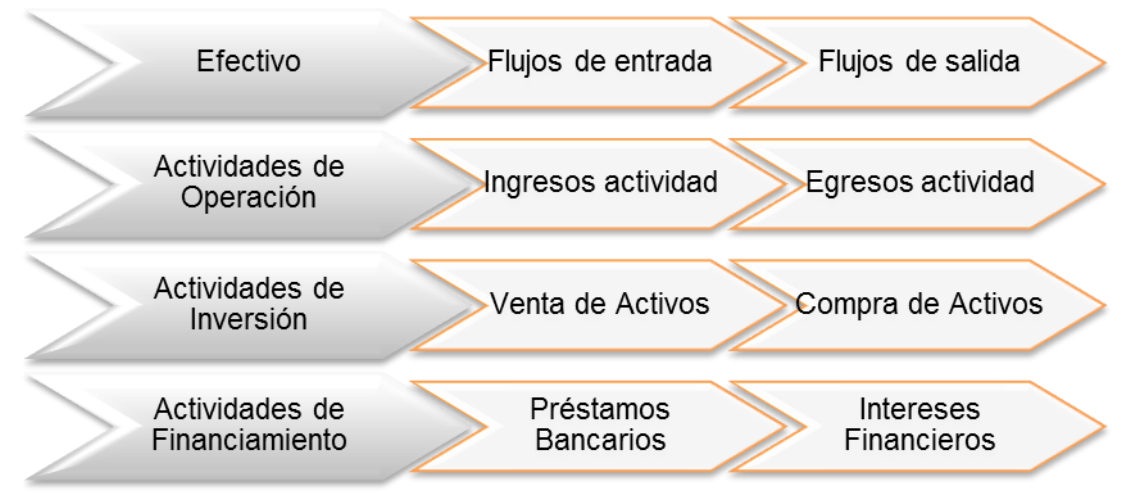

Figura 1. Clasificación de las actividades del flujo de efectivo.

Fuente: Bartley, et al. (2011).

Los flujos por actividades de operación son los rubros correspondientes a cobros y pagos relacionados con la actividad principal del negocio; y relacionados con los ingresos y gastos en la fabricación de un producto, comercialización de un bien o prestación de un servicio, así como la capacidad disponible para cubrir nuevos gastos que presente un nuevo periodo económico; por cuanto estas necesidades siempre estarán presentes buscando implementar nuevos tratamiento o procesos que aporte a mejorar la producción (Escobar-Arias, 2014). En la producción, son los rubros de producción y comercialización de la fruta, palpable en la medida de la necesidad del cuidado, mantenimiento y empaque 


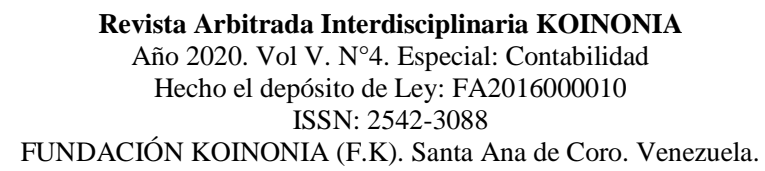

Elva Johanna Belduma-Belduma; Daniel Jacobo Andrade-Pesantez; Pablo Barahona-Vásquez

del producto, a fin de obtener una fruta de calidad apta para la exportación, evitándose en lo posible el rechazo que en ciertos ciclos acontece.

Los flujos por actividades de inversión representan los desembolsos de dinero para la adquisición de bienes que la empresa considera va a obtener a futuro una rentabilidad o flujos de efectivo que aporte al incremento del recurso económico y a la mejora de la situación financiera. Se asumen dentro de este rubro conceptos por cancelaciones para la adquisición de propiedad, planta y equipo, recaudaciones por la venta de propiedad, planta y equipo, tales como equipos para el proceso de pesada, empaque y sellado de la fruta, montacargas para facilitar el traslado de la fruta en forma segura a los diferentes contenedores evitando el maltrato y posibles accidentes en su recorrido (Arango-Álvarez, et al., 2013).

Los flujos por actividades de financiamiento se enfocan en la ganancia de recursos durante y después de ejecutar las actividades económicas por recursos externos de financiación o por los propios socios o accionistas de la empresa que repercutirá en el aumento del capital de la empresa dando valor y ganancia a la misma. Los rubros de efectivo fluctuaran incrementándose por las aportaciones de los socios o prestamos adquiridos y disminuyendo en casos de reembolsos de aportes, pagos de dividendos a socios, pagos por obligaciones contraídas a corto o largo plazo como préstamos bancarios, bonos, hipotecas dependiendo del convenio llegado para la prestación adquirida (Escobar-Arias \& Daza-Arango, 2015).

El estado de flujo de efectivo al ser un instrumento de análisis ofrece información relevante de las variaciones de efectivo que mantiene de la economía global de la empresa y ofrece al empresario un panorama adecuado para plantear decisiones que permita distribuir de manera uniforme y en orden de prioridades el destino del dinero captado en la ejecución de las actividades operativas.

\section{Toma de decisiones para la administración adecuada de los recursos}




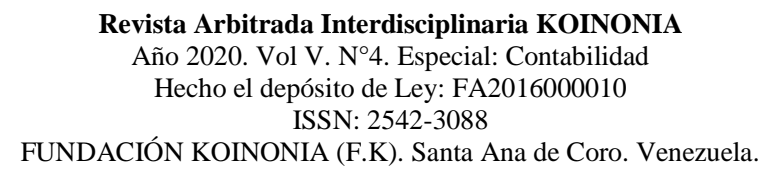

Elva Johanna Belduma-Belduma; Daniel Jacobo Andrade-Pesantez; Pablo Barahona-Vásquez

Los gerentes y propietarios de negocios tienen la ineludible tarea de decidir el futuro de las empresas que administran, a través de la debida toma de decisiones, previa evaluación y análisis de alternativas detectadas, a fin de aportar a la prosperidad o fracaso de una organización. (Balcázar-Sarmiento, et al., 2019) señalan que:

La toma de decisiones representa la llave final de todo proceso administrativo; ninguna estrategia, planificación o herramientas tienen validez sin una decisión; es decir, está vinculada a la determinación de problemas, búsqueda de soluciones, elección de las opciones más factibles y la puesta en ejecución; permitiéndose de esa manera, garantizar la viabilidad y sostenibilidad de las empresas dentro del entorno en el que se desenvuelven. (pág. 52).

En la toma de decisiones, se presenta como regla general situaciones no previstas, generándose problemas que merecen la atención inmediata de la directiva; como punto de referencia, para detectar problemas, es preciso considerar situaciones como: cambios que se presenten dentro de la estructura organizativa de la empresa, planificaciones que no están cumpliéndose dentro de los parámetros establecidos, personal que ejecuta acciones sin la aprobación de la gerencia y, la implementación de nuevas estrategias de la competencia; que en cortos periodos de tiempo repercute en la eficacia de las actividades empresariales. En tanto, las oportunidades, son las posibilidades que se presentan, para resolver problemas o cumplir los objetivos planteados por la gerencia.

(Ronda-Carracao, 2015) afirma que los sistemas actuales aportan a las decisiones con información fiable y actualizada, sin valor añadido que expanda las posibilidades. En tal sentido, las empresas requieren mejores sistemas y herramientas técnicas, para el análisis de las decisiones productivas de los negocios. (Mallo-Rodríguez \& RocafortNicolau, 2015) señalan que las empresas se enfrentan a nuevos retos cada día, lo que obliga a la gerencia a tomar decisiones con mayor frecuencia; que solucione problemas identificados en la cadena de producción, prestación de un servicio o comercialización de bienes, a fin de cumplir con el modelo de gestión estructurado.

Según los avances tecnológicos, la gestión dispone de información financiera más fiable y oportuna, aportándose competencias para decidir sobre el negocio; donde el propietario 
será quien fije al final el curso de acción a seguir; sin embargo, no siempre será capaz de encontrar la solución; por lo que es imperioso establecer un proceso de toma de decisiones eficientes que disminuya la probabilidad de errores en su ejecución.

En la figura 2 se presentan los aspectos más relevantes a considerar para la toma decisiones adecuadas.

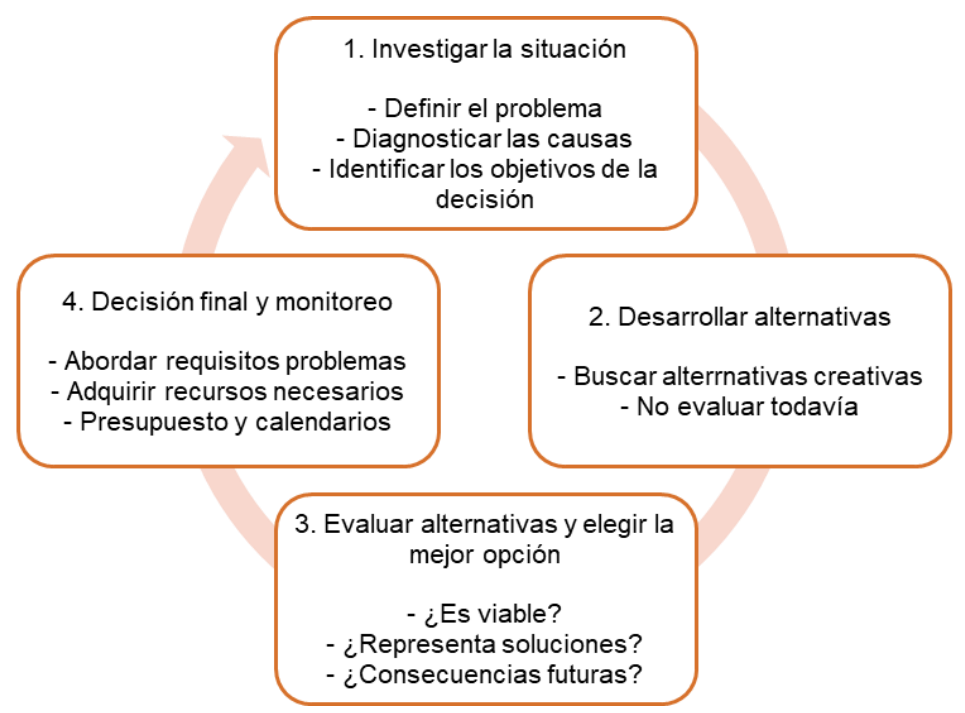

Figura 2. Representación del ciclo de toma de decisiones.

Fuente. Información obtenida de Informe Administración.

Para cumplir con las metas de la gerencia, los directivos tienen la responsabilidad de implementar estrategias de forma consecutiva, para resolver problemas suscitados dentro del entorno empresarial, ya sea por factores internos o externos que requieren soluciones inmediatas, sin afectar a la ejecución paulatina de las actividades de producción, que por lo general apelan a metodologías cuantitativas de resultados arrojados por estudios de mercado o estadísticas realizadas; a fin de lograr un crecimiento de las capacidades de producción, lo que a largo plazo permite a una unidad de negocio alcanzar la estructura e infraestructura de producción deseada y una serie de capacidades. En este sentido, las capacidades de producción son las actividades que la empresa destaca por encima de los competidores, derivadas de la infraestructura de 


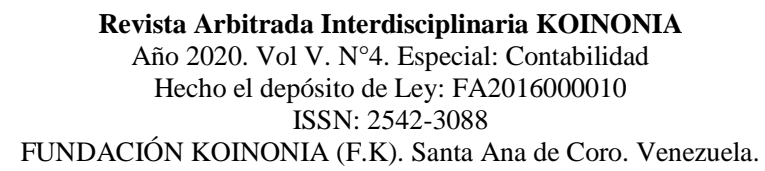

Elva Johanna Belduma-Belduma; Daniel Jacobo Andrade-Pesantez; Pablo Barahona-Vásquez

producción con la característica de ser difíciles de imitar o transferir lo que las hacen valiosas (Núñez-Segovia, et al., 2016).

La evidencia demuestra que la correcta toma de decisiones beneficia a la implementación de estrategias y medidas de control en los procesos de producción, mientras el análisis se mantenga a través de las herramientas de diagnóstico del circulante que no afecten la situación financiera del ente económico, y, sirva a los directivos en su empeño de estructurar mecanismos para el desarrollo integral de la producción.

\section{Relación de los flujos de efectivo con la toma de decisiones de producción}

Existen infinidad de procesos, modelos y herramientas que emplean las directivas de las empresas para definir las decisiones más acertadas, en beneficio del desarrollo de las empresas de producción desde un punto de vista objetivo, y de esta manera, alcanzar los objetivos propuestos. Dentro de este contexto, de acuerdo con (Amaro-Martínez, et al., 2019) y (Balcázar-Sarmiento, et al., 2019), los flujos de efectivos suministran información transparente y relevante de las entradas y salidas de efectivo, que emergen dentro del giro del negocio por situaciones puntuales, suscitadas en las actividades operativas de las empresas; y siendo flexibles en su estructura a la hora de adaptarse a las necesidades funcionales de los negocios, definidas por el sector económico al que pertenezca, y por la influencia que generan en la capacidad monetaria del Ecuador.

En su mayoría, la economía ecuatoriana depende de industrias primarias como la agricultura, el petróleo, la silvicultura y la acuicultura. sin embargo, los cambios en las tendencias del mercado global y el avance de la tecnología llevan al desarrollo de otros sectores como el textil, el procesado de alimentos y sectores de servicios (Corvo, 2019); (Elbehri, et al., 2015). En este punto destaca el sector bananero como uno de los más importantes de la economía, representando más del $25 \%$ del total de las exportaciones agrícolas y el $10 \%$ de las exportaciones totales, siendo el segundo ingreso más importante después del petróleo y, uno de los sectores más dinámicos con las mayores 


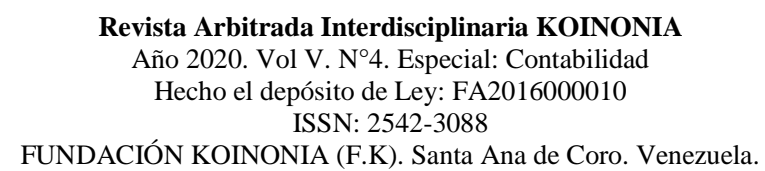

Elva Johanna Belduma-Belduma; Daniel Jacobo Andrade-Pesantez; Pablo Barahona-Vásquez

tasas de crecimiento de hasta el $12 \%$ en el mercado; donde las grandes plantaciones bananeras del mundo se encuentran en zonas tropicales de países en desarrollo.

En El Ecuador el banano se cultiva en regiones húmedas con temperaturas altas, entre $18^{\circ}$ y $30^{\circ} \mathrm{C}$. Casi todo el banano producido en Ecuador es de las provincias de El Oro, Guayas o Los Ríos y, según el registro del Ministerio de Agricultura y Ganadería (MAG), el Ecuador tiene alrededor de 162.236 hectáreas sembradas de banano y cuenta con 4.473 productores de la fruta, como se aprecia en la tabla 1.

\section{Tabla 1.}

Cuadro distributivo del sector bananero.

\begin{tabular}{ccc}
\hline $\begin{array}{c}\text { Distribución por tamaño } \\
\text { de hectáreas }\end{array}$ & $\begin{array}{c}\text { Hectáreas } \\
\text { Sembradas }\end{array}$ & $\begin{array}{c}\text { Número de } \\
\text { Productores }\end{array}$ \\
\hline $0-30$ (Pequeños) & 35.685 & 3480 \\
$>30<100$ (Medianos) & 57.486 & 800 \\
100 o más (Grandes) & 69.063 & 193 \\
TOTAL & 162.236 & 4.473 \\
\hline
\end{tabular}

Fuente: Ministerio de Comercio Exterior (2017).

Las empresas bananeras son consecuentes en su aporte a la economía y funcionales con el análisis del ciclo de producción del banano, para mejorar y desarrollar nuevas estrategias funcionales que genere más rentas a los productores. "La competitividad exige que las empresas busquen mecanismos que avalen la eficiencia y la eficacia de las operaciones, así como el cumplimiento oportuno ante los organismos de control, y la acertada toma de decisiones para contar con información auténtica" (Murillo-Apolo, et al., 2019). Los rubros de entradas y salidas del efectivo en las empresas del sector bananero constan de las partidas de operación, inversión y financiación, originadas en un ejercicio económico, donde la base principal para la aplicación de la herramienta, consiste en determinar los procesos que componen el cultivo del banano, necesarios en la evaluación y análisis respectivo de los gastos empleados en las fases de producción, comercialización y logística de la fruta, así como, determinar la capacidad del negocio 


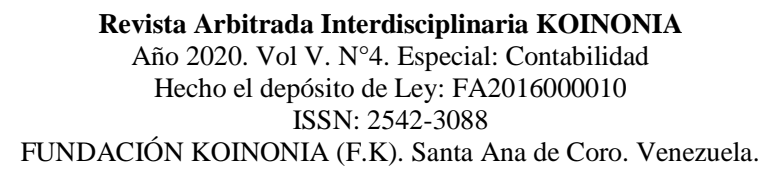

Elva Johanna Belduma-Belduma; Daniel Jacobo Andrade-Pesantez; Pablo Barahona-Vásquez

para acceder a préstamos o inversiones que aporten a su credibilidad y expansión en el mercado (Escobar-Arias \& Daza-Arango, 2015); (Saavedra-García, \& Loé-Uribe, 2018). Uno de los sectores con más solvencia en el mercado nacional e internacional es el bananero, puesto que representa una de las mayores fuentes de ingresos para el país; de ahí se sustenta la importancia de contar con un esquema de flujo de efectivo y su aplicación constante para mantener al productor informado (Murillo-Apolo, et al., 2019). En su mayoría, los empresarios que están al frente de estos negocios tienen poco o nulo conocimiento de la contabilidad, así como; los costos invertidos en la producción, enfrentándose a situaciones que por desconocimiento se vuelven críticas como: Pago al personal, uso de tarjetas empresariales en función del cupo disponible, rentabilidad para comprometerse en nuevos proyectos, establecer lineamientos para ejecutar medidas sostenibles en el negocio y el desarrollo del flujo.

El flujo de efectivo es una herramienta complementaria a los estados financieros que elaboran de forma anual o mensual dentro del giro del negocio, de gran valor para el productor por cuanto está diseñada a las necesidades y exigencias establecidas; socializado y aprobado por el empresario en las medidas de sus requerimientos a la fecha; presenta de forma oportuna y detallada todos los gastos en las actividades de operación, inversión y financiación, su valor inicial y final, carteras y obligaciones actualizadas para analizar y considerar si está en las condiciones adecuadas para invertir dinero que acrecienten su valor en el mercado.

Bajo este contexto, el presente trabajo tuvo por objeto elaborar una guía para el diseño del flujo de efectivo que aporte a la toma de decisiones de producción en las empresas del sector bananero del Cantón el Guabo.

\section{MÉTODO}

Para la realización de esta investigación se adoptó un tipo descriptiva con diseño de campo no experimental transversal, que proporcionó información relacionada con el flujo de efectivo y la toma de decisión, obtenida en un tiempo establecido. Así mismo se apoyó 


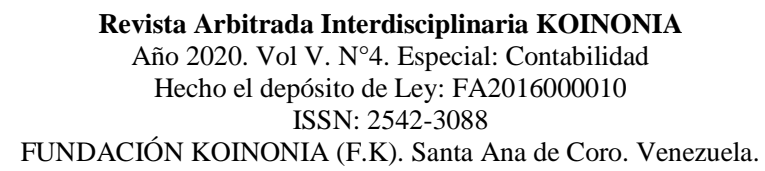

Elva Johanna Belduma-Belduma; Daniel Jacobo Andrade-Pesantez; Pablo Barahona-Vásquez

en la revisión documental con la finalidad de analizar las teorías referenciales al tema, en función de indagación bibliográfica, sobre documentación financiera, criterios, experiencias en el medio y análisis de sucesos pasados en empresas bananeras, a lo largo del tiempo y sus consecuencias relevantes en el entorno económico.

Los métodos utilizados en esta investigación fueron el deductivo que sirvió para determinar el cumplimiento de los parámetros establecidos por la norma en la elaboración del flujo de efectivo, y el acceso a datos significativos que aporten a la resolución de la problemática planteada en el estudio. Así mismo, se empleó el método analítico-sintético que permitió verificar la repercusión de la información financiera contenida en los flujos de efectivo en las decisiones tomadas por la gerencia de las empresas; como la comprensibilidad y análisis realizados por los profesionales de contabilidad en el cumplimiento de la normativa.

Para la realización de este estudio se aplicó el muestreo no probabilístico por conveniencia, considerando los criterios de ubicación geográfica y disponibilidad de tiempo. En este sentido, la muestra empleada para esta investigación tuvo un alcance de 20 empresas del total de la población que conforma las empresas del sector bananero del Cantón El Guabo.

Para el propósito de la cuantificación de la información se empleó como técnica de recolección de datos la encuesta y cuestionario de varias alternativas, analizándose os datos recopilados, la estadística descriptiva para abordar temas relevantes que valoren los indicadores determinados en el problema de estudio y aportar al análisis de los resultados; en este caso puntual realizadas a profesionales de contabilidad y gerentes expertos en el área bananera.

\section{RESULTADOS}

Para evaluar la contribución del flujo de efectivo en la toma de decisiones de producción de las empresas del sector bananero se utilizó como apoyo a la investigación la participación de representantes legales y profesionales del área contable, a fin de 


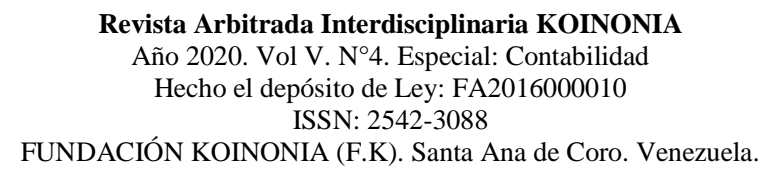

Elva Johanna Belduma-Belduma; Daniel Jacobo Andrade-Pesantez; Pablo Barahona-Vásquez

solventar la incertidumbre permanente que mantienen los empresarios, con relación al mecanismo de apoyo que representa una herramienta financiera en la administración correcta del efectivo, sus equivalentes, garantizar aperturas a inversiones, financiamientos, en beneficio del ente económico. La estructura técnica de la encuesta, se conformó de 19 preguntas cerradas con opciones múltiples, la información suministrada constituye el criterio que tienen los profesionales con relación a la problemática.

\section{Área Contable-Financiera}

Los resultados obtenidos de la primera pregunta de la encuesta demostraron que el $95 \%$ de los profesionales del área contable consideran que es importante la elaboración del estado de flujo de efectivo, ya que permite saber los movimientos generales del efectivo dentro de la bananera; sin embargo, el $95 \%$ de los contadores manifestaron que la elaboración del estado de flujo de efectivo se realiza para cumplir con la normativa vigente del país y tan sólo un $5 \%$ la considera como una herramienta útil de análisis financiero, para determinar el flujo del efectivo dentro del giro del negocio. De acuerdo con los resultados, el $85 \%$ de la muestra indicó la complejidad y vacíos que tienen los profesionales en la actualidad para interpretar la NIC 7 y aplicarla en la elaboración del estado financiero, y solo el 15\% de los participantes manifestó no tener problemas para comprender la ley.

Con relación a la información que considera el profesional contable al momento de la elaboración del informe financiero más de la mitad de la muestra, es decir, el $65 \%$ señaló que los inconvenientes que encuentran en la elaboración del flujo se centra en la información de fuente y uso del efectivo, por cuanto tienen un vacío de los rubros que componen cada una de las actividades que se clasifican en la estructura del estado financiero; seguido por un $20 \%$ que no tiene problemas, y el $15 \%$ que no es aplicable. Estos resultados sugieren, en síntesis, que la problemática que surge se debe a que no hay un acertado reconocimiento de la naturaleza de las cuentas o rubros, como 


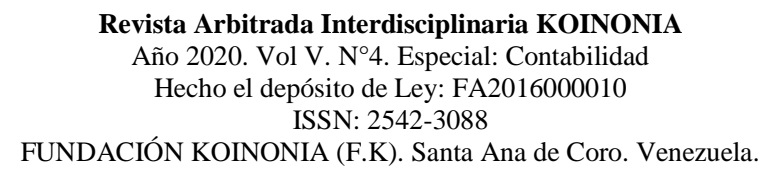

Elva Johanna Belduma-Belduma; Daniel Jacobo Andrade-Pesantez; Pablo Barahona-Vásquez

consecuencia de la falta de capacitación en los temas relevantes al estado financiero como se apreció en datos anteriores; o la falta de atención para comprender el mecanismo que se emplea en la elaboración del flujo de efectivo.

El estudio evidencio la falta de análisis e interpretación del estado de flujo de efectivo que aseveraron el $60 \%$ de los profesionales; mientras un $40 \%$ consideran este aspecto relevante para la administración de los recursos de la empresa; lo que revela que la información que suministra el flujo de efectivo no se aprovecha en el buen desempeño de las actividades operativas del negocio; en cambio se elaboran de forma mecánica para cumplir con la norma vigente y exigencia de los entes reguladores; y, tampoco se emplea para tomar decisiones que encaminen el desarrollo de la producción.

Los datos revelaron que el $65 \%$ de los contadores consideran al estado de flujo de efectivo como una herramienta útil para la administración del efectivo que contradice la falta de análisis e interpretación del informe financiero por parte de contabilidad que manifestaron con anterioridad; mientras el 35\% desconoce las ventajas del flujo de efectivo en la administración de los recursos financieros. En este sentido, los resultados de la investigación reafirman la importancia de destacar la utilidad y beneficios que otorga la información relevante del estado financiero frente al sistema bancario nacional, para crear un ambiente de confianza a nuevas oportunidades de inversiones y financiamientos.

\section{Área de Gerencia}

Del total de participantes que respondieron la encuesta el $100 \%$ reportaron que no emplean la información del estado de flujo de efectivo para tomar decisiones relacionadas con la producción; que reafirma la poca importancia que muestran en la información sustancial que reporta de los movimientos del efectivo para planear estrategias fiables que garanticen su efectividad.

Contar con una herramienta de análisis es fundamental como apoyo para las decisiones de la gerencia y evitar caer en el desconocimiento del destino de determinados rubros 


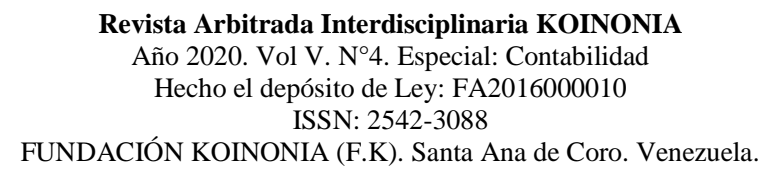

Elva Johanna Belduma-Belduma; Daniel Jacobo Andrade-Pesantez; Pablo Barahona-Vásquez

dentro de las actividades económicas del negocio. En tal sentido, el $45 \%$ de los profesionales emplean los informes de producción como herramienta para el análisis de la capacidad adquisitiva de las empresas bananeras, por el criterio que manejan los propietarios sobre la eficiencia de la información suministrada con relación al rendimiento de la producción, el 25\% emplea el Estado de Resultados Integrales, así como, un 15\% emplea el flujo de efectivo al determinar la capacidad económica que tiene la empresa para asumir obligaciones.

Considerando los aspectos antes citados, los directivos implementan instrumentos que consideran relevantes para cumplir los objetivos propuestos, y que demuestre la capacidad o conocimiento para ejercer el cargo. En concordancia a lo señalado, el resultado de la encuesta mostró que el $85 \%$ de la muestra a veces cumple con los objetivos propuestos, mientras el 15\% siempre cumple con las metas trazadas. En ese sentido, es natural considerar que las herramientas financieras empleadas no brindan información relevante que garantice el buen desempeño de las áreas productivas de las bananeras y optar por nuevas opciones más fiables.

Un $75 \%$ de los participantes afirmó que consideran sustancial la información del flujo de efectivo para la toma de decisiones y solo un 5\% manifestó lo contrario; lo que demuestra que saben de las bondades que otorgan a la administración adecuada de los recursos para plantear estrategias futuras. Sin embargo, los encuestados respondieron que el $75 \%$ desconoce el proceso de análisis e interpretación de los resultados para la correcta toma de decisiones que acreciente su valor en el sistema financiero y visionar a mejoras de infraestructura o adquisición de propiedades para nuevas plantaciones, mientras un $5 \%$ lo considera innecesario.

En general los resultados indicaron que los profesionales conocen de las ventajas que proporciona el estado de flujo de efectivo como instrumento financiero para la buena marcha de un negocio, sin embargo, tiene cierto grado de complejidad la preparación, análisis e interpretación del mismo, debido a la aplicación inadecuada u omisión de la norma, por cuanto, carecen de uniformidad en los criterios sobre los lineamientos 0 
procesos a seguir para la preparación, lo que dificulta también la interpretación y análisis adecuado.

\section{PROPUESTA}

Los resultados proporcionan soporte concluyente de la relación entre los flujos de efectivo y la toma de decisiones que responde a la problemática planteada y muestra la relevancia que tiene dentro de una empresa para conocer con exactitud los montos de efectivo administrados por la gerencia. Así mismo, permite vislumbrar oportunidades a largo plazo y cubrir los requerimientos de la empresa que se intercalan en periodos de tiempo, por diferentes factores que afecta a toda la producción, diseminándose a las áreas de administración y logística.

Para una mejor comprensión de las bondades del flujo de efectivo se determinó necesario elaborar una guía para su diseño que permita al empresario una comprensión global de todos los rubros económicos que se manejan en cada una de las etapas del ciclo de producción del banano, y la factibilidad de sus resultados en la determinación de alternativas y posterior análisis que faculte a la gerencia para la correcta toma de decisiones. Bajo este contexto, en la figura 6 se detallan los componentes que contendrá la guía y servirán para dar una visión más clara de los aspectos importantes del proceso. 

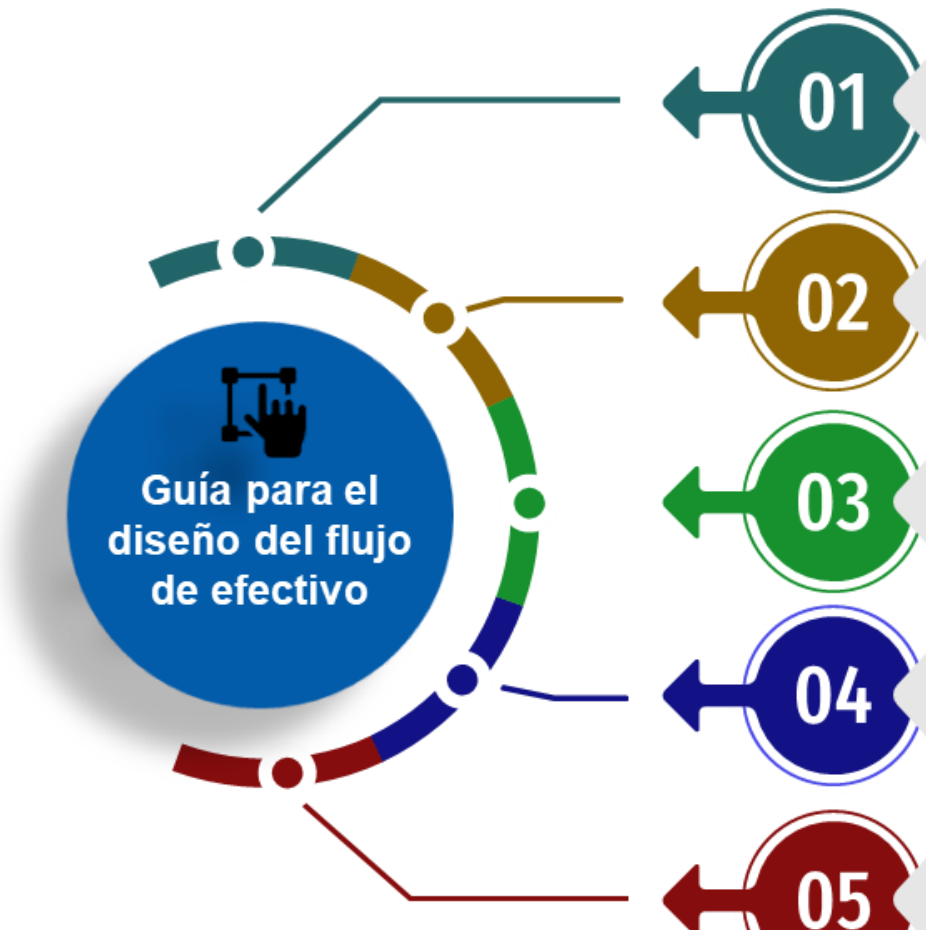

Introducción y alcance

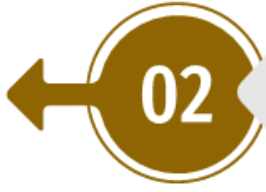

Reconocimiento de las partidas del efectivo

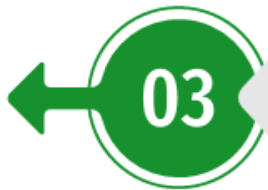

Segregación de las actividades

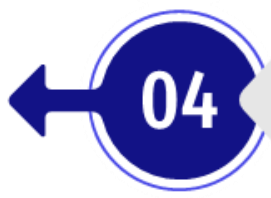

Esquema de información financiera

(11)

\section{5}

Análisis para la toma de decisiones

Figura 3. Esquema de propuesta de guía para el diseño del flujo de efectivo.

\section{GUÍA PARA EL DISEÑO DEL FLUJO DE EFECTIVO PARA EMPRESAS BANANERAS Introducción y alcance}

El sector bananero en el entorno económico representa uno de los rubros más fuertes para la economía del Ecuador y es considerado dentro de los grupos más sólidos en la actualidad; conformado por un gran número de empresas distribuidas en compañías y personas naturales, donde radica la necesidad de mantenerse proactivos en el mercado. Sin embargo, existen falencias palpables en ciertos entes económicos que a pesar de su consolidación en el mercado les dificulta administrar sus recursos, debido a la ausencia de instrumentos de análisis.

La normativa vigente en el país, en la NIC 7 suministra a los usuarios de los estados financieros una herramienta eficaz que aporta información histórica del flujo de efectivo y sus equivalentes, para evaluar la capacidad adquisitiva de la empresa con miras a 


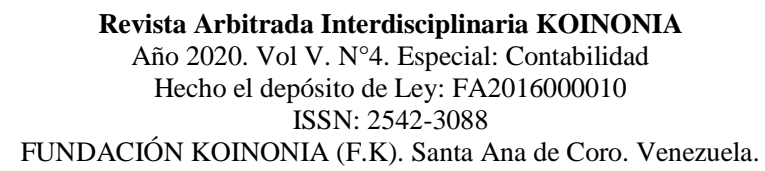

Elva Johanna Belduma-Belduma; Daniel Jacobo Andrade-Pesantez; Pablo Barahona-Vásquez

plantearse metas de progreso y desarrollo del negocio. En este sentido, su desarrollo implica una constante para el área contable, por la dificultad en su ejecución y a la vez la información fiable que aporta y no se considera en la mayoría de las empresas.

El propósito de esta guía es orientar de forma práctica la elaboración del flujo de efectivo y enfatizar la importancia que representa en las decisiones de producción la revelación de la información de los estados financieros.

El contenido de la guía para el diseño del flujo de efectivo es de carácter académico que presenta aspectos relevantes a considerarse por los responsables del tratamiento de la información financiera en su ejecución.

\section{Reconocimiento de las partidas del efectivo}

El efectivo y sus equivalentes, es una partida contable definida en el estado de situación financiera que representa el dinero disponible que tiene la empresa, así como, los activos de corto plazo que pueden ser convertidos de forma inmediata. Se deben reconocer las partidas que cumplan con la definición de entradas y salidas del efectivo.

Las entradas del efectivo implican todos los ingresos relacionados con la actividad económica de la empresa, que aumentan el efectivo en un periodo determinado, y están relacionados con su naturaleza contable focalizadas en el área de producción, al ser el área más predominante en la actividad de banano; que pueden ser:

Venta de banano. - El precio oficial de la caja de banano fijado por el MAG para el año 2020 es de $\$ 6.40$ que representa la principal fuente de ingresos del productor que se obtiene por semana de acuerdo con el número de cajas procesadas en los embarques

Venta de rechazo de banano. - La fruta que no pasa el proceso de calidad es depositada en un apartado que se destina a las industrias locales para su posterior proceso en la obtención de subproductos.

Intereses por pólizas. - El excedente de efectivo se deposita en pólizas a fin de aprovecharlo y obtener réditos en periodos definidos por el productor sean estos mensual, trimestral, semestral o anual. 


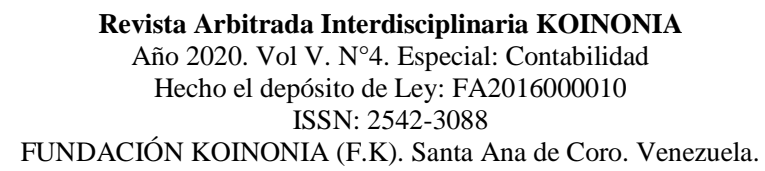

Elva Johanna Belduma-Belduma; Daniel Jacobo Andrade-Pesantez; Pablo Barahona-Vásquez

Las salidas del efectivo representan todos los gastos relacionados con la actividad operativa de la empresa que disminuyen el efectivo en un tiempo establecido. En este sentido, las principales fuentes de salida de efectivo corresponden:

Nóminas. - Es el registro financiero que elabora la empresa, relacionado con los jornales y salario del área de producción, administración y logística.

Beneficios sociales. - Representa el décimo tercero, décimo cuarto que la empresa debe cancelar al empleado en fechas específicas estipuladas por el Código de Trabajo.

Utilidades. - Es la participación resultante del $15 \%$ de la utilidad operacional de la empresa para los trabajadores y se cancelan hasta el 15 de abril de cada año, estipulada en el Código de Trabajo.

Impuestos fiscales. - Las empresas tienen la obligación de efectuar declaraciones mensuales correspondientes a las retenciones en la fuente, así como, del impuesto al valor agregado de acuerdo con el noveno dígito del calendario publicado en las páginas del Servicio de Rentas Internas.

Seguridad social. - La afiliación de los trabajadores implica el pago mensual de las planillas normales, fondos de reserva, extensión conyugal, préstamos quirografarios e hipotecarios según sea el caso.

Proveedores. - Desembolsos de dinero por la compra de productos fitosanitarios para el mantenimiento y tratamiento de producción relacionadas con plagas, bacterias 0 manchas que atacan a las plantas productoras; así como; para el abastecimiento de suministros para el departamento de administración.

Préstamos bancarios. - Corresponden a deudas adquiridas con instituciones financieras con el ánimo de mejoras para la empresa y deben cubrir cada mes con el interés correspondiente pactado. 


\section{Segregación de las actividades}

En este caso específico, la estructura del flujo estará dado por el método directo, puesto que presenta los movimientos del efectivo de forma ordenada de los principales cobros y pagos que mantiene la empresa, y que consolida la información del estado de resultados integrales para la planificación y elaboración de presupuestos, así como, para una mejor comprensión y análisis del efectivo que se administra. En consecuencia, la estructura del flujo de efectivo, su composición pondera del impacto que suponen las actividades en la posición financiera del negocio, determinadas por tres actividades claves que deben ser identificadas para el posterior análisis y elaboración del estado financiero que son:

De operación. - Los flujos de efectivo procedentes de esta actividad son un elemento sustancial en la medida que ha generado fondos líquidos para cubrir obligaciones internas y externas, así como, mantener la capacidad de producción, pagar dividendos y ejecutar inversiones sin necesidad de recurrir a fuentes de financiamiento. Estas actividades proceden de las operaciones y otros sucesos relevantes dentro de la actividad económica de la empresa, como se ilustra en la tabla 2.

\section{Tabla 2.}

Actividades de Operación.

\begin{tabular}{r|l}
\hline \multicolumn{1}{c}{ Código } & \multicolumn{1}{c}{ Cuenta } \\
\hline 95010101 & Cobros procedentes de la venta de banano \\
95010102 & Cobros procedentes de otros ingresos de actividades ordinarias \\
95010201 & Pagos a proveedores por el suministro de bienes y servicios \\
95010203 & Pagos a y por cuenta de los empleados \\
950103 & Dividendos pagados \\
950104 & Dividendos recibidos \\
950105 & Intereses pagados \\
950106 & Intereses recibidos \\
\hline
\end{tabular}


De inversión. - Los flujos de efectivo procedentes de esta actividad son los desembolsos de dinero en la adquisición de recursos que van a producir beneficios futuros; como la compra de lotes de tierra para plantaciones, vehículos para el traslado de la fruta al puerto y construcción de empacadora; cómo se aprecia en la tabla 3.

\section{Tabla 3.}

Actividades de inversión.

\begin{tabular}{ll}
\hline Código & \multicolumn{1}{c}{ Cuenta } \\
\hline 950201 & Efectivos procedentes de la venta de acciones \\
950205 & Pagos para adquirir acciones o instrumentos de venta \\
950208 & Importes procedentes por la venta de propiedad, planta y equipo \\
950209 & Adquisiciones de propiedad, planta y equipo \\
\hline
\end{tabular}

De Financiación. - Representan las actividades que suponen un cambio en el tamaño y composición de los capitales y préstamos adquiridos por la empresa, para ganar mayor valor en el mercado, así como, para la adquisición de maquinaria y equipo para cumplir con el ciclo de producción de la fruta en cada una de sus etapas, como se destaca en la tabla 4.

\section{Tabla 4.}

Actividades de Financiación.

\begin{tabular}{ll}
\hline Código & \multicolumn{1}{c}{ Cuenta } \\
\hline 950301 & Aporte en efectivo por aumento de capital \\
950302 & Financiamiento por emisión de títulos valores \\
950304 & Financiamiento por préstamos a largo plazo \\
950305 & Pagos préstamos \\
\hline
\end{tabular}




\section{Esquema de información financiera}

La información que aborda el flujo de efectivo constará de la consolidación de los rubros presentados en el estado de situación financiera y el estado de resultados integrales consolidados para determinar los desembolsos de dinero que se han efectuado en un periodo económico y determinar el flujo neto de efectivo que mantiene la empresa al inicio de un periodo dado. En efecto, los estados financieros constituyen una representación gráfica de la situación económica que atraviesa el negocio, a fin de decidir en el curso de las operaciones y a su vez conocer la gestión realizada con el recurso otorgado.

Una vez reconocido los elementos del flujo, se procede al análisis de la fuente de información de los estados financieros en los pasos siguientes:

a) Realizar un comparativo de los estados financieros correspondientes al ejercicio anterior, con los balances del presente año; a fin de cotejar el monto empleado en las actividades realizadas y la relevancia que representa el giro del negocio.

b) Establecer las variaciones positivas o negativas, a partir de la comparación de los saldos de las cuentas contables de los estados financieros que dependerán de la recaudación de recursos o pago de las obligaciones pendientes.

c) Consolidar los valores en la plantilla del flujo de efectivo con las consideraciones del caso, a fin de distribuir los recursos de acuerdo con el origen de cada uno de ellos, debiéndose consolidar en el apartado de incremento o disminución neto del efectivo y equivalente del efectivo con el fondo disponible que reporta el estado de situación financiera (Ver Figura 4). 


\begin{tabular}{|c|c|c|}
\hline \multicolumn{3}{|l|}{ COMPAÑÍA DE PRODUCTORES DE BANANO S.A. } \\
\hline ESTADO DE FLUJOS DE EFECTIVO POR EL MÉTODO DIRECTO & & SALDOS \\
\hline AL 31 DE DICIEMBRE DEL 2019 & & BALANCE \\
\hline & CODIGO & (En US\$) \\
\hline $\begin{array}{l}\text { INCREMENTO NETO (DISMINUCIÓN) EN EL EFECTIVO Y EQUIVALENTES AL DE EFECTIVO, ANTES DEL EFECTO } \\
\text { DE LOS CAMBIOS EN LA TASA DE CAMBIO }\end{array}$ & 95 & 32111,32 \\
\hline FLUJOS DE EFECTIVO PROCEDENTES DE (UTILIZADOS EN) ACTIVIDADES DE OPERACIÓN & 9501 & $-100785,37$ \\
\hline Clases de cobros por actividades de operación & 950101 & 849905,44 \\
\hline Cobros procedentes de las ventas de bienes y prestación de servicios & 95010101 & 845393,89 \\
\hline Cobros procedentes de regalías, cuotas, comisiones y otros ingresos de actividades ordinarias & 95010102 & 0 \\
\hline Otros cobros por actividades de operación & 95010105 & 4511,55 \\
\hline Clases de pagos por actvidades de operación & 950102 & $-871917,29$ \\
\hline Pagos a proveedores por el suministro de bienes y servicios & 95010201 & $-803769,83$ \\
\hline Pagos procedentes de contratos mantenidos para intermediación o para negociar & 95010202 & 0 \\
\hline Pagos a y por cuenta de los empleados & 95010203 & $-68147,46$ \\
\hline Otros pagos por actividades de operación & 95010205 & 0 \\
\hline Dividendos pagados & 950103 & 0 \\
\hline Dividendos recibidos & 950104 & 0 \\
\hline Intereses pagados & 950105 & 0 \\
\hline Intereses recibidos & 950106 & 0 \\
\hline Impuestos a las ganancias pagados & 950107 & $-405,74$ \\
\hline Otras entradas (salidas) de efectivo & 950108 & $-78367,78$ \\
\hline FLUJOS DE EFECTIVO PROCEDENTES DE (UTILIZADOS EN) ACTIVIDADES DE INVERSIÓN & 9502 & -446 \\
\hline Efectivo procedentes de la venta de acciones en subsidiarias u otros negocios & 950201 & 0 \\
\hline Otros pagos para adquirir acciones o instrumentos de deuda de otras entidades & 950205 & 0 \\
\hline Importes procedentes por la venta de propiedades, planta y equipo & 950208 & 0 \\
\hline Adquisiciones de propiedades, planta y equipo & 950209 & -446 \\
\hline Otras entradas (salidas) de efectivo & 950221 & 0 \\
\hline FLUJOS DE EFECTIVO PROCEDENTES DE (UTILIZADOS EN) ACTIVIDADES DE FINANCIACIÓN & 9503 & 133342,69 \\
\hline Aporte en efectivo por aumento de capital & 950301 & 0 \\
\hline Financiamiento por emisión de títulos valores & 950302 & 0 \\
\hline Pagos por adquirir o rescatar las acciones de la entidad & 950303 & 0 \\
\hline Financiación por préstamos a largo plazo & 950304 & 133342,69 \\
\hline Pagos de préstamos & 950305 & 0 \\
\hline Intereses recibidos & 950309 & 0 \\
\hline Otras entradas (salidas) de efectivo & 950310 & 0 \\
\hline EFECTOS DE LA VARIACION EN LA TASA DE CAMBIO SOBRE EL EFECTIVO Y EQUIVALENTES AL DE EFECTIVO & 9504 & 0 \\
\hline Efectos de la variación en la tasa de cambio sobre el efectivo y equivalentes al efectivo & 950401 & 0 \\
\hline INCREMENTO (DISMINUCIÓN) NETO DE EFECTIVO Y EQUIVALENTES AL EFECTIVO & 9505 & 32111,32 \\
\hline EFECTIVO Y EQUIVALENTES AL EFECTIVO AL PRINCIPIO DEL PERIODO & 9506 & 1353,67 \\
\hline EFECTIVO Y EQUIVALENTES AL EFECTIVO AL FINAL DEL PERIODO & 9507 & 33464,99 \\
\hline
\end{tabular}

Figura 4. Modelo del estado de flujo de efectivo.

\section{Análisis para la toma de decisiones}

Una vez finalizada la elaboración del flujo de efectivo, la disponibilidad de esta herramienta sirve como base a la gerencia en el análisis integral de las posibilidades que le ofrecen los resultados revelados, a considerar:

Situación financiera de la bananera: Se distinguirá la distribución de los recursos en cada una de las operaciones generadas por la empresa y el impacto que tiene determinados rubros en la situación financiera del negocio, permitiendo al productor evaluar si los flujos establecidos surgieron de la necesidad imperecedera de la producción o fueron otros los factores que influyeron en su desembolso. 


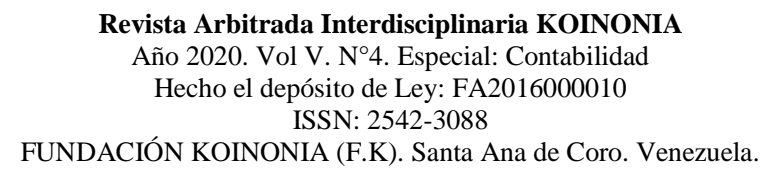

Elva Johanna Belduma-Belduma; Daniel Jacobo Andrade-Pesantez; Pablo Barahona-Vásquez

Implementación de estrategias. - A partir de la información revelada por el flujo de efectivo la gerencia tiene la facultad para determinar la capacidad de la empresa en el tiempo, evaluar y plantear alternativas proactivas para frenar los problemas que surjan en lo posterior; así como, administrar los recursos de manera adecuada para no reincidir en las mismas afectaciones; y en su lugar disponer de recursos para nuevos proyectos, adquisición de equipos y maquinarias que aporten a optimizar resultados.

La toma de decisiones óptima dependerá del buen uso de las herramientas disponibles y la gestión correcta de los recursos que revelan, para mantenerse proactivo en el mercado y en lo posible lograr un crecimiento financiero, que permita acceder a inversiones sin la necesidad de endeudamientos externos.

\section{CONCLUSIONES}

La literatura existente ha evidenciado el aporte sustancial del flujo de efectivo para suministrar información fiable de la situación financiera de la empresa, a fin de evaluar y plantear alternativas que aporten al buen funcionamiento y desarrollo de las actividades operacionales.

Como ya se mencionó en la revisión de la literatura científica para un análisis correcto de la capacidad económica de la empresa es imprescindible seguir un proceso que garantice a la gerencia decisiones mejores planteadas, con proyecciones de mejoras en el entorno empresarial y aporten al rendimiento financiero del negocio a largo plazo.

El hallazgo más interesante que se extrajo del estudio fue el hecho que las empresas del sector bananero, presentan el flujo de efectivo de acuerdo con la normativa legal NIC 7 , sin embargo, no emplean este estado financiero como instrumento de evaluación para la toma de decisiones a pesar de la información que representa dentro del ámbito económico.

Los resultados son consistentes con la necesidad de aplicar una herramienta eficaz que evalué el disponible de las bananeras y considerar que el flujo de efectivo es el estado financiero más viable como apoyo del departamento contable en sus funciones de 
reportar a los directivos, información constante de las variaciones y movimientos del circulante que maneja la empresa.

La elaboración de la guía para el diseño del flujo de efectivo en las empresas bananeras es una herramienta, para orientar y esclarecer las dudas planteadas por el departamento contable que incluye información necesaria de la estructura integral del estado financiero y debe considerarse para evitar errores en los saldos del efectivo.

La guía propuesta del estudio cumple con las consideraciones de la Superintendencia de Compañías y normas contables que facilita al profesional de contabilidad una secuencia de pasos para elaborar con facilidad el flujo de efectivo y revelar información que faculte a la gerencia al momento de tomar decisiones para la administración adecuada de los recursos.

\section{FINANCIAMIENTO}

No monetario.

\section{AGRADECIMIENTO}

A las empresas del sector bananero del Cantón El Guabo, Ecuador; por su disponibilidad en el desarrollo de la investigación.

\section{REFERENCIAS}

Amaro-Martínez, D, Acevedo-Suárez, J, \& Amaro-Martínez, D. (2019). La integración de las finanzas al flujo logístico. Aplicación: proceso de alimentación [.The integration of finance to the logistics flow. Application: feeding process]. Ingeniería Industrial, 40(1), 97-108.

Andrade-Valenzuela, P, Toscano-Ruiz, D, \& Parrales-Higuera, M. (2018). El estado de flujo de efectivo, herramienta de gestión para evaluar la capacidad de los administradores. [The cash flow statement, management tool to assess the ability of administrators]. Revista FADMI: Administración y Tecnología, 2(2); 11-16. 


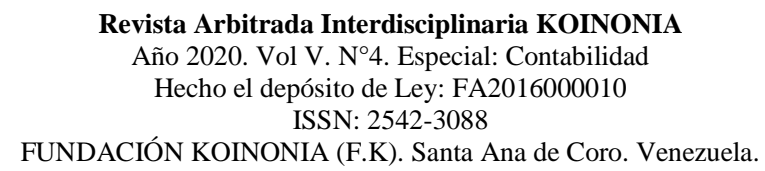

Elva Johanna Belduma-Belduma; Daniel Jacobo Andrade-Pesantez; Pablo Barahona-Vásquez

Apolo-Ordoñez, G., Narváez-Zurita, C., \& Erazo-Álvarez, J. (2019). El Control interno como herramienta de apoyo a la gestión financiera del Gobierno Autónomo Descentralizado Municipal Zaruma. [Internal Control as a tool to support the financial management of the Zaruma Municipal Decentralized Autonomous Government]. CIENCIAMATRIA, 5(1), https://doi.org/10.35381/cm.v5i1.280

551-577.

Arango-Álvarez, R. D., Arias-Vargas, F. J., Escobar-Arias, G. E., \& Molina-Osorio, A. (2013). Relaciones entre los flujos de efectivo de las empresas de Caldas y el crecimiento económico regional para el sector industrial durante el período de 2002 a 2010. [Relations between the cash flows of the Caldas companies and the regional economic growth]. Revista Lasallista de Investigación, 10(1); 75-90.

Balcázar-Sarmiento, B. M., Erazo-Álvarez, J. C., \& Narváez-Zurita, C. I. (2019). Herramientas de contabilidad gerencial para la toma de decisiones financieras Cía. Ltda. de la ciudad de Cuenca. [Management accounting tools for financial decision making Cía. Ltda. Of the city of Cuenca]. Visionario Digital, 3(2.2), 50-80. https://doi.org/10.33262/visionariodigital.v3i2.2.602

Bartley, D., Block, S., \& Hirt, G. (2011). Fundamentos de Administración Financiera. Edición 14. [The flow statement Fundamentals of Financial Administration. Edition 14]. Mexico: McGRAW-HILL.

Corvo, H. (2019). Sectores económicos del Ecuador: Características principales [Economic sectors of Ecuador: Main characteristics]. Recuperado de https://n9.cl/xkva

Duque-Sánchez, A. (2015). Estado de flujos de efectivo: aplicación de razonamientos algebraicos y de la NIC 7. [Statement of cash flows: application of algebraic reasoning and IAS 7]. Cuadernos De Contabilidad, 16(40). https://doi.org/10.11144/Javeriana.cc16-40.efea

Elbehri, A., G. Calberto, C. Staver, A. Hospido, L. Roibas, D. Skully, P. Siles, J. Arguello, I. Sotomayor, y A. Bustamante. (2015). Cambio climático y sostenibilidad del banano en el Ecuador: Evaluación de impacto y directrices de política. Organización de las Naciones Unidas para la Alimentación y la Agricultura (FAO), Roma, Italia. 
Escobar-Arias G. E. (2104). Flujos de efectivo y entorno económico en las empresas de servicios de Risaralda 20022011. [Cash flows and economic environment in Risaralda service companies 2002-2011]. Revista Finanzas y Política Económica, 6(1), 141-158.

Escobar-Arias, G. (2016). Crecimiento Económico como determinante de los flujos de efectivo para las empresas del sector industrial de Caldas - Colombia, durante el periodo 1995 - 2013. [Economic Growth as a determinant of cash flows for companies in the industrial sector of Caldas - Colombia, during the period 1995 2013]. ÁNFORA, 23(41), 179-201. https://doi.org/10.30854/anf.v23.n41.2016.146

Escobar-Arias, G. (2018). Crecimiento económico y flujos de efectivo: aplicación al sector industrial de los departamentos de Antioquia y Valle del Cauca (Colombia). [Economic growth and cash flows: application to the industrial sector of the departments of Antioquia and Valle del Cauca (Colombia)]. ÁNFORA, 25(44), 91111. https://doi.org/10.30854/anf.v25.n44.2018.400

Escobar-Arias, G. E., \& Daza-Arango, J. W. (2015). Relaciones entre los flujos de efectivo de las empresas del departamento de Risaralda y el crecimiento económico regional para el sector agropecuario, durante el periodo comprendido de los años 2002 a 2011. [Relationships between the cash flows of companies in the department of Risaralda and regional economic growth for the agricultural sector, during the period from 2002 to 2011]. Revista RETO, 3(3), 105-115.

Funes, Y. (2012). Reseña "Herramientas de planeación financiera para las Pymes" de María Luisa Saavedra García. [Review "Financial planning tools for SMEs" by María Luisa Saavedra García]. Actualidad Contable Faces, 15(24),75-80.

Gonzalo-Angulo, J. A. (2003). El estado de flujo de efectivo y el estado de cambios en el patrimonio neto: origen, contenido y utilidad. [The statement of cash flow and the statement of changes in equity: origin, content and profit]. Boletín de estudios económicos, LVIII(178); 41-73.

International Financial Reporting Standard (IFRS). (2007). NIC 7 Estado de Flujos de Efectivo. [IAS 7 - Statement of Cash Flows]. Recuperado de https://cutt.ly/EonYO4U

Mallo-Rodríguez, C., \& Rocafort-Nicolau, A. (2015). Contabilidad de dirección para la toma de decisiones: Contabilidad de gestión y de costes. [Management accounting for decision-making: Management and cost accounting]. Barcelona: Profit Editorial.

Ministerio de Comercio Exterior. (2017). Informe Sector Bananero Ecuatoriano [Ecuadorian Banana Sector Report]. Recuperado de https://n9.cl/banate 
Murillo-Apolo, L., Narváez-Zurita, C., \& Erazo-Álvarez, J. (2019). Sistema de control interno con enfoque en la ISO 9001: 2015 en la bananera Monterrey. [Internal control system focused on ISO 9001: 2015 in the Monterrey banana company]. Revista Arbitrada Interdisciplinaria Koinonía, 4(2), 241-264. http://dx.doi.org/10.35381/r.k.v4i2.474

Saavedra-García M. L., \& Loé-Uribe, J. (2018). Flujo de efectivo para las pymes: una propuesta para los sectores automotor y de tecnologías de la información en México. [Cash Flow for SMEs: A Proposal for the Automotive and Information Technology Sectors in Mexico]. Revista Finanzas y Política Económica, 10(2), 287-308. https://doi.org/10.14718/revfinanzpolitecon.2018.10.2.3

Núñez-Segovia, J, Godoy-Garvs, J, \& Pérez-Pozo, L. (2016). Determinación de restricciones de capacidad de producción en proceso de obtención de cobre. [Determination of production capacity constraints in the process of obtaining copper]. Ingeniare. Revista chilena de ingeniería, 24(Especial), 4959. https://dx.doi.org/10.4067/S0718-33052016000500007

Ortíz-Anaya, H., \& Ortíz-Niño, D. A. (2018). Flujo de caja y proyeciones financieras con análisis de riesgo. [Cash flow and financial projections with risk analysis]. 3a edición Colombia: Universidad Externado de Colombia. DGP Editores S.A.S.

Rodríguez-Masero, N., \& López-Manjón, J. D. (2016). El flujo de caja como determinante de la estructura financiera de las empresas españolas. Un análisis en tiempos de crisis. [Cash Flow as Determinant of Financial Structure of Spanish Firms. Analysis in a Crisis Period]. Revista De Métodos Cuantitativos Para La Economía Y La Empresa, 21; 141-159.

Rodríguez-Masero, Natividad, \& López-Manjón, Jesús D. (2016). El Flujo de caja como determinante de la estructura financiera de las empresas españolas. Un análisis en tiempos de crisis. [Cash flow as a determinant of the financial structure of Spanish companies. An analysis in times of crisis]. Revista de Métodos Cuantitativos para la Economía y la Empresa, 21; 141-159.

Ronda-Carracao, M. Á. (2015). UF1883 - Instalación de sistemas ERP-CRM. [UF1883 Installation of ERP-CRM systems]. España: Editorial Elearning. 
Saavedra-García M. L., \& Loé-Uribe J. (2018). Flujo de efectivo para las pymes: una propuesta para los sectores automotor y de tecnologías de la información en México. [Cash Flow for SMEs: A Proposal for the Automotive and Information Technology Sectors in Mexico]. Revista Finanzas y Política Económica, 10(2), 287-308. https://doi.org/10.14718/revfinanzpolitecon.2018.10.2.3

Tovar-Posso, M., \& Muñoz-Martínez, I. (2018). Metodología para valoración financiera de PYMES colombianas utilizando flujos de efectivo. [Methodology for financial valuation of Colombian SMEs using cash flows]. Civilizar: Ciencias Sociales $Y$ Humanas, 18(35), https://doi.org/10.22518/usergioa/jour/ccsh/2018.2/a11

139-162.

Urgal-González, B., \& García-Vázquez, J. M. (2006). Decisiones de producción, capacidades de producción y prioridades competitivas. Un estudio aplicado al sector del metal en España. [Production decisions, production capacities and competitive priorities. A study applied to the metal sector in Spain]. Investigaciones Europeas de Dirección y Economía de la Empresa, 12(3),133-149.

Van-Horne, J., \& Wachowicz, J. (2002). Fundamentos de administración financiera. Undécima edición. [Fundamentals of financial administration. Eleventh edition]. México: Pearson Prentice Hall.

Vargas-Soto, R. (2007). Estado de flujo de efectivo [Cash flow statement]. InterSedes: Revista de las Sedes Regionales, VIII(14),111-136. 